\title{
Closed tank pneumatic press application to improve Sauvignon Blanc wine quality and nutraceutical properties
}

\author{
Pietro Catania, ${ }^{1}$ Filippa Bono, ${ }^{2}$ Claudio De Pasquale, ${ }^{1}$ Mariangela Vallone ${ }^{1}$ \\ ${ }^{1}$ Department of Agricultural, Food and Forest Sciences; ${ }^{2}$ Department of Economic Business and Statistical Sciences, \\ University of Palermo, Italy
}

\begin{abstract}
The machines used in the winemaking process directly affect the quality of wine and its nutraceutical properties. Grapes' pressing is a very important step in winemaking as it may promote the presence and/or absence of enzymatic processes on the must, leading to the creation of different products in terms of chemical composition, starting from the same grapes. The aim of the study was to compare two different pressing systems of Sauvignon Blanc grapes using an innovative pneumatic discontinuous closed tank press in two operating modes: the traditional pressing mode in presence of oxygen and the inert pressing mode, performed through grapes pressing under inert gas with nitrogen recovery. Chemical composition of musts and wines was analysed. Pressing under inert atmosphere caused an increase in total polyphenols; total acidity values in musts raised up denoting a very favourable environment for the development of the aromatic component of the future wine. The absorbance measured at $420 \mathrm{~nm}$, an index of total browning reaction of foods, was significantly lower in the wine coming from inert pressing. Principal component analysis application allowed extracting composite quality indicators of must for evaluating the effectiveness of the inert pressing procedure. Results are encouraging and open up new research prospective with the aim of applying innovative techniques to improve the quality of the final product.
\end{abstract}

Correspondence: Mariangela Vallone, Department of Agricultural, Food and Forest Sciences, University of Palermo, viale delle Scienze ed. 4, 90128 Palermo, Italy.

Tel.: +39.91.23865609.

E-mail: mariangela.vallone@unipa.it

Key words: Inert pressing; must and wine; principal component analysis; winemaking.

Acknowledgements: the authors are grateful to Puleo srl, Italy and to its General Manager Dr. Matteo Curatolo, for providing the pneumatic press used in the tests.

Received for publication: 3 August 2018

Accepted for publication: 17 June 2019.

${ }^{\circ}$ Copyright: the Author(s), 2019

Licensee PAGEPress, Italy

Journal of Agricultural Engineering 2019; L:896

doi:10.4081/jae.2019.896

This article is distributed under the terms of the Creative Commons Attribution Noncommercial License (by-nc 4.0) which permits any noncommercial use, distribution, and reproduction in any medium, provided the original author(s) and source are credited.

\section{Introduction}

Innovative technologies play a very important role in the winemaking process as they can influence chemical, sensory and nutritional quality of wine (Catania et al., 2016). The development of fruity scents is a matter of great interest especially for white grapes. The molecules responsible for such scents in wines are volatile thiols that have been identified in Sauvignon Blanc wines (Tominaga et al., 1998). The biogenesis of these molecules is believed to be related to the glutathione metabolism. Mattivi et al. (2012) demonstrated that glutathione is preserved with soft pressing conditions and by paying particular care to avoiding any oxidative mechanisms. Patel et al. (2010) studied the impact of juice press fractions on the content of varietal thiols in Sauvignon Blanc wines at laboratory scale obtaining a less than half concentrations of molecules contributing to fruity character in the must coming from pressed juice compared to wines made from free run juices. Moreover, the amount of pressure applied during grape pressing affects the extraction of varietal aromas located in the skin (Maggu et al., 2007) confirming that the pressing step plays a very important role among all the winemaking procedures especially for Sauvignon Blanc wines as for other white wines (Ferreira-Lima et al., 2016). In fact, grapes' pressing represents a very important phase in winemaking since it can promote the presence and/or absence of enzymatic processes endogenous to the juice, leading to the creation of different products in terms of chemical composition.

In the last decades, the production of white wines has been oriented towards processes performed under conditions of low oxygen level (Boselli et al., 2010). Oxygen, in fact, can influence the composition and quality of wine drastically, either positively or negatively. Oxygen exposure naturally occurs during grapes mechanical harvest and early winemaking procedures as destemming, crushing and pressing. Grape juice can be protected against enzymatic oxidation by sulphur dioxide and the presence of glutathione. The use of $\mathrm{SO}_{2}$ as an anti-oxidant dates back to the early $18^{\text {th }}$ century and the protection of wine from unwanted oxidative spoilage has been recognised (Ribéreau-Gayon et al., 2000). The effect of oxygen during fermentation on wine composition or sensory proprieties changes with grapes cultivar. In Boselli et al. (2010) the effect of nitrogen gas on three white grapes varieties (Chardonnay, Grechetto and Orvieto) was observed, obtaining the strongest protective effect of nitrogen on phenolics in Chardonnay and Grechetto musts. The authors showed that nitrogen gas is therefore particularly recommended not only in positive pressure, but also for vacuum-pressing of white grapes containing high levels of catechin or gallic acid due to early harvest or peculiar varietal composition. The effect of reductive pressing on colour, glutathione, total polyphenols and catechins was studied by Motta et al. (2014) on four Italian white grapes cultivars, obtaining a low- 
ering of the main quality parameters of the musts processed in contact with air during pressing. Pressing under nitrogen on Sauvignon Blanc grapes was applied by Pons et al. (2015), obtaining an increase in glutathione concentration when low pressure was applied $(<1$ bar $)$.

Interest in wine press innovation suggests the opportunity of carrying out real-scale experiments to assess the effect that different types of grapes presses may have on the must and, consequently, on the quality of wine.

In our study we considered a closed tank pneumatic press applied on Sauvignon Blanc grapes used in two different modes, the traditional air pressing mode and the inert pressing mode. The aim of the study was to investigate if the two different pressing modes influence must and wine characteristics and to identify the possible improvement of wines obtained through innovative machines.

\section{Materials and methods}

\section{Experimental tests}

The trials were carried out during the vintage 2016 in the province of Trapani (Sicily, Italy). The Sauvignon Blanc grapes came from a 8-year vineyard set at $420 \mathrm{~m}$ above sea level, 4 hectares wide. The vineyard was hedgerow trained with 3333 plants/ha (planting layout $2.50 \times 1.20 \mathrm{~m}$ ) and Guyot pruning; the 2016 production was about 0.80 tons per hectare. Sauvignon Blanc is one of the most widespread and famous grapes variety. It is a white berry variety with a high concentration of indirect primary aromas that become perceptible in the wine only after the biochemical activities of fermentation (Marais, 1998).

Grapes were manual harvested in the third decade of August, at their optimal ripening stage, and immediately placed in plastic perforated boxes to allow airing, prevent crushing and abrasion of the berries which can trigger fermentation processes and microbiological contamination with consequent loss of cellular juices and product quality.

The must composition was determined in three different times during winemaking $\left(29^{\text {th }}\right.$ August, $1^{\text {st }}$ September and $3^{\text {th }}$ September), in triplicate samples each. The analyses on wine were performed at the end of fermentation.

\section{Pneumatic press used for grapes processing}

The pneumatic discontinuous closed tank press is actually the most used machine in quality wine making. It is a hollow AISI 304 stainless steel cylinder rotating around a horizontal axis, with a side-mounted flexible food grade PVC membrane inside opposed to a wide wall of longitudinal holed channels that allow must draining. The destemmed-crushed grapes are loaded through a side opening, provided with a sealed door, or an axial load valve. After loading, pressure is exerted on the other side of the membrane by compressed air to extract the must. The operation is repeated several times progressively increasing pressure, alternating with a retracted membrane rotation phase, in order to mix the progressively drier marc. At the end of the extraction phase, skins and grape seeds are unloaded through the side opening.

In our study a Prexa N30 pneumatic press (Puleo Srl, Italy) was used to process the grapes (capacity of $1900 / 2500 \mathrm{~kg}$ ) using a sequential pressing cycle. The initial rotations and drainage lasted $40 \mathrm{~min}$, during which the pressure was $<0.2$ bar. In the subsequent 110 min cycle, pressure was gradually raised to 2 bar including pressing and rotations.
The machine is equipped with the patented Vortex system that allows grapes pressing under inert gas with nitrogen recovery. The idea of using inert gas in pressure during working cycle, guarantees a continuous draining action with promising results in product quality as stated by many authors (Boselli et al., 2010; Motta et al., 2014; Pons et al., 2015). A further advantage is the immediate extraction of the must from the cylinder to the storage tank thanks to the Vortex created inside the press. During grapes processing, the inert gas is continuously filtered to be purified by any pollution due to unwanted parts that can be carried by the gas itself.

The wine press used in the tests can work in two operating modes: the traditional air pressing mode (AP) and the nitrogen pressing mode (NP), according to the classification adopted in Motta et al. (2014).

In our study, the AP mode is the traditional pneumatic press with closed tank. Grape must is extracted from the inner draining channels and comes out from nozzles to an external collection tray. In the NP mode, grapes contact with air is minimised and the grapes are processed in presence of nitrogen (Figure 1).

The machine is equipped with a vertical tank compliant with Pressure Equipment Directive (PED) 2014/68/EU, vitrified inside for food use. Besides allowing the continuous transfer of inert gas, it has the function of maintaining and storing nitrogen to be reused (Figure 2).

The other technological phases of grapes processing were carried out in the same way for both batches processed in AP and NP modes.

\section{Analytical determination in musts and wines}

Analytical determination in musts and wines were performed by Foss Integrator WineScan ${ }^{\mathrm{TM}}$, (FOSS Italia S.p.A.). The must and wine determinations were alcohol [\%/vol], density [g/L], sugar $[\mathrm{g} / \mathrm{L}], \mathrm{pH}$, total acidity $[\mathrm{g} / \mathrm{L}]$, volatile acidity $[\mathrm{g} / \mathrm{L}]$, malic acid $[\mathrm{g} / \mathrm{L}]$, citric acid $[\mathrm{g} / \mathrm{L}]$, tartaric acid $[\mathrm{g} / \mathrm{L}]$, potassium $[\mathrm{g} / \mathrm{L}]$, polyphenols $[\mathrm{mg} / \mathrm{L}]$, ashes $[\mathrm{g} / \mathrm{L}]$, RAN (readily assimilable nitrogen) $[\mathrm{g} / \mathrm{L}]$, gluconic acid $[\mathrm{g} / \mathrm{L}]$, methanol $[\mathrm{g} / \mathrm{L}], \mathrm{CO}_{2}[\mathrm{~g} / \mathrm{L}]$, absorbance at 420, 520 and $620 \mathrm{~nm}$ for wine, catechins [mg/L]. The observations were performed in triplicate for each time during winemaking, respectively in $29^{\text {th }}$ August, $1^{\text {th }}$ September and $3^{\text {th }}$ September, 2016.

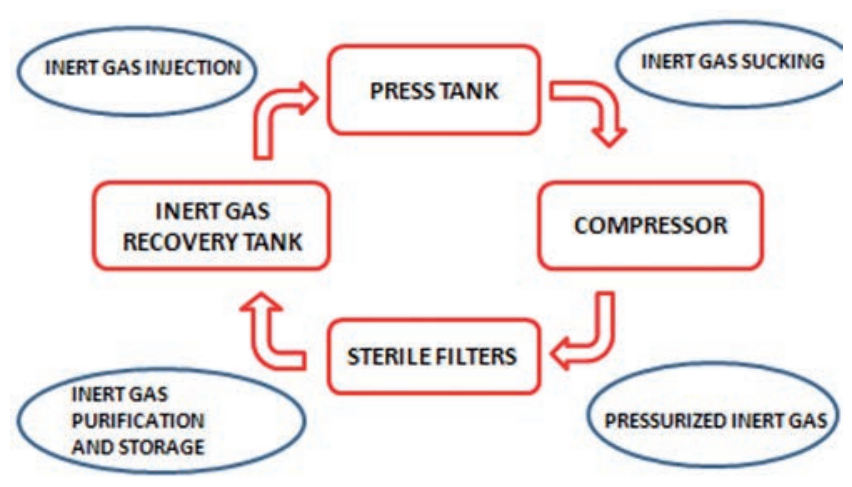

Figure 1. Vortex system used in the nitrogen pressing operating mode. The inert gas is pressurised by the compressor, filtered and sent to a recovery tank, then injected into the press and, finally, recycled and sent back to the compressor to start a new cycle. 
Esters and higher molecular weight alcohols were also investigated, identified and determined in wine samples by using headspace solid-phase microextraction (HS-SPME) coupled to gas chromatography-mass spectrometry (GC-MS) techniques, which provide a simple and green extraction and concentration analytical method. In order to identify and subsequently determine each compound, experimental sequence of standard organic compounds were injected by using the same extraction procedure. Moreover Kovats Indices (KI), on the base of linear hydrocarbon standard injection, were considered for the identification. Indices (KI) were derived considering the retention time normalised to the adjacently eluted $n$-alkanes. On the above base KI are independent from the analytical procedures and therefore useful for the identification of unknown compounds.

The gas-chromatographic analyses were run on a HewlettPackard 5890 GC system interfaced with a HP 5973 quadrupole mass spectrometer. A HP5-MS column was used (5\% diphenyl $95 \%$ dimethylpolysiloxane $30 \mathrm{~m} \times 0.2 \mathrm{~mm}, 0.25 \mu \mathrm{m}$ film, J \& W Scientific, Folsom CA, USA). Ultra-high-purity helium (Praxair, Cleveland, $\mathrm{OH}$ ) was the carrier gas. Water and oxygen traps were installed on the carrier gas lines was employed. The column temperature was held at $40^{\circ} \mathrm{C}$ for $15 \mathrm{~min}$ and then was increased to $220^{\circ} \mathrm{C}$ at $1^{\circ} \mathrm{C} / \mathrm{min}$. The carrier gas (helium) flow rate was $1 \mathrm{~mL}$ $\mathrm{min}^{-1}$. The spectra were recorded at an ionisation voltage of 70 eVand an ion source temperature of $220^{\circ} \mathrm{C}$ (De Pasquale et al., 2006). Samples were analysed by HS-SPME-GC-MS method with a PDMS-CAR-DVB fibre (Supelco). The fibre was manually inserted in a $\mathrm{GC}$ inlet port equipped with a specific glass liner for SPME injection $\left(0.75 \mathrm{~mm}\right.$ i.d.) Fibres were desorbed at $250^{\circ} \mathrm{C}$ in splitless mode for $1 \mathrm{~min}$ into gas chromatograph inlet. Sample components were verified by comparison of the mass spectral data with those of authentic reference compounds. When standards were not available, the components were identified by mass spectrum matching using the NIST05 mass spectral library collection.

\section{Statistical analysis}

The ANOVA models were considered in order to test if there were differences in the two pressing mode (AP, air pressing and NP, nitrogen pressing) in must characteristics during time (three different times during winemaking were considered). To test if there were statistical differences in wine characteristics, $t$-test was performed. Differences were considered significant at 5\% level of significance for t-test. Principal component analysis was applied in order to reduce the variable data setting of must and extract composite quality indicators. The amount of each compound was considered as the dependent variable of the measured experimental parameters. According to Kaiser (1960) and the scree plot (a plot of the eigenvalues shown in decreasing order) proposed by Cattell (1966) we retained only components whose eigenvalues are higher than 1. All the statistical analyses were carried out using Stata, version 14.1.

\section{Results and discussion}

Must chemical composition obtained by using the two pressing procedures was affected by the different treatment. The ANOVA was applied to test the existence of statistically significant differences between AP and NP and also during must fermentation, considering the three times when analytical determinations were performed. In particular, statistically significant differences were obtained for density, $\mathrm{pH}$, tartaric acid and gluconic acid with respect to time. Statistical differences between the two pressing modes are represented in Table 1. Alcohol, volatile acidity, and $\mathrm{CO}_{2}$ concentration levels were higher in AP, while the levels of total acidity, malic acid, potassium, polyphenols, ashes, RAN were higher in NP. Also Motta et al. (2014) found no differences between the must obtained in presence of oxygen and the must pro-

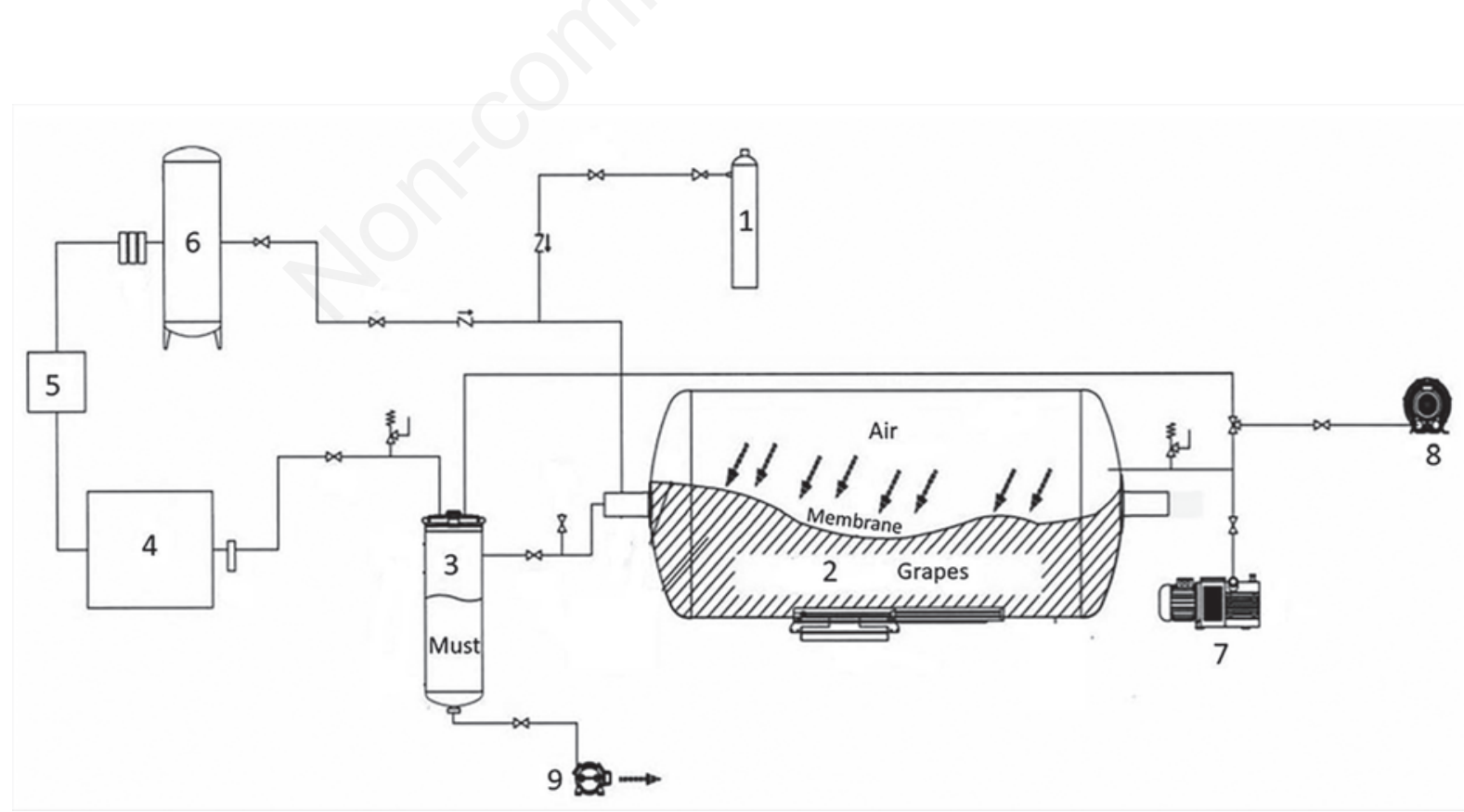

Figure 2. Pneumatic press with nitrogen recovery. Initial nitrogen injection cycle inside the press 1-2 (1: nitrogen cylinder, 2: nitrogen inside the press in contact with grapes); nitrogen recovery, filtration, storage and reuse phase with the Vortex System 3-6 (3: nitrogen recovery from the press, 4: compressor, 5: dryer, 6: recovered nitrogen storage for re-use); pump for compression (7); vacuum pump (8); pump for the transfer of the extracted must (9). 
cessed in inert atmosphere in total sugars, titratable acidity and $\mathrm{pH}$. They also noted that the use of the pneumatic press in normal atmosphere caused a $30 \%$ decrease in total polyphenols of Moscato bianco musts, compared to a $43 \%$ decrease obtained in our study for Sauvignon Blanc musts. The decrease was cultivar dependent and was also influenced by the vintage.

Total acidity rises up from 5.27 to $6.78 \mathrm{~g} / \mathrm{L}$ from AP to NP showing a significant increase $(+29 \%)$ denoting a very favourable environment (for total acidity values above $6 \mathrm{~g} / \mathrm{L}$ ) for the development of esters, or the aromatic component of the future wine. On the contrary, Pons et al. (2015) obtained no differences in total acidity between the musts obtained by pressing with and without nitrogen. Malic acid significantly increased from AP to NP, too, contrary to the previous authors.

Potassium in the samples treated with nitrogen was higher than those treated in air-pressing, this is a positive effect for the purpose of tartaric stabilisation.

RAN concentration was considerably higher in the samples treated in inert atmosphere; this was certainly favoured by the insertion of nitrogen inside the pressing tank. In qualitative terms, these concentrations are very important to guarantee the nutrition of the yeasts that ensures the correct development of the alcoholic fermentation.

The t-test performed at 5\% level of significance showed statistically significant differences in almost all the analytical determinations of wines obtained with the two pressing modes (Table 2). In particular, total extract, $\mathrm{pH}$, total acidity, malic acid, citric acid, ashes, polyphenols, calcium, copper and sulphates obtained with the NP pressing system were significantly higher than AP. Volatile acidity was significantly lower in NP, and this results represents a positive effect of inert pressing as the presence of oxygen favours the formation of acetic acid. In fact, acetic acid is the major constituent of volatile acidity; all the acids that make up volatile acidity give complexity to the aroma of wine, but if the acetic acid concentration exceeds the perception threshold, it could be considered a problem in the wine. A similar result was obtained by Pons et al.
(2015) comparing volatile acidity of Sauvignon Blanc wines obtained by pressing with and without nitrogen.

Malic acid value of wine obtained from grapes pressed in NP mode doubled compared to the value of the wine resulting from the use of the machine in AP mode, while Pons et al. (2015) did not observe any difference between inert and normal pressing for this parameter. Preserving malic acid in white wines is a key factor to guarantee their freshness and youth (Hartwig and McDaniel, 1995).

The absorbance measured at $420 \mathrm{~nm}$ (OD420, that is optical density at $420 \mathrm{~nm}$ ) is considered as an index of total browning reaction of foods. In the wine obtained processing the grapes with inert gas, OD420 was significantly lower than the value of the wine obtained processing grapes in normal atmosphere $(-44 \%)$ that is near to the result obtained by Boselli et al. (2010) on Orvieto grapes, an Italian cultivar.

When the must was protected from oxygen, catechins obtained in the corresponding wine have a notable increase, raising from $11.6 \mathrm{mg} / \mathrm{L}$ in the AP pressing mode to $14.6 \mathrm{mg} / \mathrm{L}$ in NP. This result is in agreement with Motta et al. (2014).

Acetaldehyde is an extremely reactive volatile substance, responsible for the so-called oxidised odour in wines, reminiscent of that emanated from the rotten apple. It is formed starting from the oxidation of ethanol caused by hydrogen peroxide, which is obtained, by the oxidation of polyphenols. High values of acetaldehyde, therefore, can give defects to white wines, so the result obtained in NP mode is more desirable than that of AN mode.

The presence of polyphenols is very important as antioxidant and preservatives. The NP pressing mode shows a polyphenols concentration in the must samples, $468.70 \mathrm{mg} \mathrm{L}^{-1}$, that was significantly higher than the AP pressing mode, $269.92 \mathrm{mg} \mathrm{L}^{-1}$. Looking at the wines, a $10 \%$ increase in polyphenols was obtained from AP to NP. This remarkable result on nutraceutical properties of Sauvignon Blanc wine product, helps to increase the values of wine form economical and marketing point of views and has a key role in the prevention of cardiovascular disease and other chronic

Table 1. Descriptive statistics of must composition in air pressing and nitrogen pressing modes.

\begin{tabular}{|c|c|c|c|c|c|c|c|c|c|}
\hline \multirow[b]{2}{*}{ Variable } & \multicolumn{4}{|c|}{$\mathbf{A P}$} & \multicolumn{5}{|c|}{ NP } \\
\hline & Mean & Std. Dev. & Min & Max & Mean & Std. Dev. & Min & $\operatorname{Max}$ & \\
\hline Alcohol [\%/vol] & 10.84 & 1.51 & 8.90 & 12.55 & 10.35 & 1.88 & 7.89 & 12.12 & $*$ \\
\hline Density [g/L] & 1.01 & 0.02 & 0.97 & 1.04 & 1.01 & 0.02 & 0.98 & 1.04 & ns \\
\hline Sugar [g/L] & 39.58 & 22.85 & 15.85 & 69.00 & 44.14 & 29.27 & 15.98 & 82.49 & ns \\
\hline $\mathrm{pH}$ & 3.41 & 0.05 & 3.33 & 3.51 & 3.38 & 0.04 & 3.33 & 3.44 & ns \\
\hline Volatile acidity [g/L] & 0.21 & 0.01 & 0.18 & 0.22 & 0.18 & 0.01 & 0.16 & 0.20 & $*$ \\
\hline Total acidity $[\mathrm{g} / \mathrm{L}]$ & 5.27 & 0.15 & 5.42 & 5.89 & 6.78 & 0.11 & 6.62 & 6.96 & * \\
\hline Malic acid [g/L] & 2.66 & 0.15 & 2.50 & 2.99 & 3.52 & 0.13 & 3.35 & 3.73 & $*$ \\
\hline Citric acid [g/L] & 0.29 & 0.04 & 0.24 & 0.36 & 0.31 & 0.04 & 0.26 & 0.37 & ns \\
\hline Tartaric acid [g/L] & 2.57 & 0.15 & 2.35 & 2.78 & 2.47 & 0.16 & 2.20 & 2.66 & ns \\
\hline Potassium [g/L] & 988.63 & 52.54 & 926.37 & 1073.00 & 1153.22 & 28.74 & 1107.60 & 1189.99 & * \\
\hline Polyphenols [mg/L] & 269.92 & 32.15 & 221.19 & 321.16 & 468.70 & 56.88 & 380.00 & 528.53 & $*$ \\
\hline Ashes $[\mathrm{g} / \mathrm{L}]$ & 2.38 & 0.10 & 2.24 & 2.56 & 2.64 & 0.11 & 2.50 & 2.80 & * \\
\hline RAN $[g / L]$ & 28.18 & 17.50 & 6.52 & 50.00 & 80.18 & 18.10 & 58.68 & 103.90 & $*$ \\
\hline Gluconic acid [g/L] & 2.54 & 0.13 & 2.34 & 2.72 & 2.56 & 0.16 & 2.30 & 2.80 & ns \\
\hline Methanol [g/L] & 0.45 & 0.51 & 0.10 & 1.15 & 0.14 & 0.02 & 0.12 & 0.19 & ns \\
\hline $\mathrm{CO}_{2}[\mathrm{~g} / \mathrm{L}]$ & 1418.73 & 156.31 & 1236.00 & 1638.56 & 1251.59 & 261.60 & 948.00 & 1615.00 & $*$ \\
\hline
\end{tabular}

AP, air pressing; NP, nitrogen pressing. *Indicates significance at $\mathrm{P} \leq 0.01$ and ns indicates not significant. 
pathologies, including cancer (Cordova and Sumpio, 2009). Polyphenols are medicine and phenols usually constitute the major compositional difference between red and white wines. Numerous epidemiological studies found that a regular and moderate consumption correlates inversely with vascular disease and mortality, therefore such a notable increase of polyphenols can be a strength for white wines marketing.

Concentrations of the ethyl esters of branched acids were affected by the procedure AP and NP. In particular, the level of acids obtained in NP mode is favourable to the development of ester profile (Table 3) that determines the aromatic component of wines. The ethyl esters include a short-chain alcohol group (ethanol) and a longer-chain acid group (such as medium- to longchain fatty acids). There are many factors that influence the types and quantities of esters present and although all of them are not positive contributors. As a group, they are a major constituent of wine. Ethyl esters have a strong influence on a wine's aroma with reported aroma characteristics that include respectively for AP and NP, Hexanoic acid, ethyl ester 8.73 and $8.81 \%$, with fruity, strawberry, green apple, Octanoic acid, ethyl ester 35.20 and $33.10 \%$ with sweet, fruity, ripe fruit, burned and Decanoic and Dodecanoic acid, ethyl ester with beer characteristics, oily, fruity and floral character, $34.56,34.92 \%$ and $7.15,6.29 \%$, respectively. The aroma profile appears fully representative of cultivar Sauvignon Blanc in the two procedures, AP and NP.

Many analytical parameters describe as the AP and NP technological procedures may affect the production of Sauvignon Blanc
Table 3. Chemical compounds and their relative abundances on wine in air pressing and nitrogen pressing modes.

\begin{tabular}{|c|c|c|c|}
\hline Kovats index & & & Compounds \\
\hline & $\mathbf{A P}$ & NP & \\
\hline 697 & 2.39 & 2.37 & 1-Butanol, 3-methyl- \\
\hline 743 & 0.12 & 0.20 & 2,3-Butanediol \\
\hline 820 & 5.13 & 8.45 & 1-Butanol, 3-methyl-, acetate \\
\hline 984 & 8.73 & 8.81 & Hexanoic acid, ethyl ester \\
\hline 996 & 1.77 & 1.94 & Acetic acid, hexyl ester \\
\hline 1158 & 1.17 & 0.79 & Octanoic Acid \\
\hline 1183 & 35.20 & 33.10 & Octanoic acid, ethyl ester \\
\hline 1223 & 0.57 & 0.42 & Acetic acid, 2-phenylethyl ester \\
\hline 1372 & 0.82 & 0.58 & n-Decanoic acid \\
\hline 1389 & 1.25 & 0.97 & Ethyl 9-decenoate \\
\hline 1391 & 34.56 & 34.92 & Decanoic acid, ethyl ester \\
\hline 1466 & 0.43 & 0.65 & Octanoic acid, 3-methylbutyl ester \\
\hline 1580 & 7.15 & 6.29 & Dodecanoic acid, ethyl ester \\
\hline 1615 & 0.20 & 0.24 & Pentadecanoic acid, 3-methylbutyl ester \\
\hline 1726 & 0.22 & 0.12 & Tetradecanoic acid, ethyl ester \\
\hline 1968 & 0.29 & 0.22 & Hexadecanoic acid, ethyl ester \\
\hline
\end{tabular}

$\mathrm{AP}$, air pressing; $\mathrm{NP}$, nitrogen pressing.

Table 2. Descriptive statistics of wine composition in air pressing and nitrogen pressing modes.

\begin{tabular}{|c|c|c|c|c|c|c|c|c|c|}
\hline \multirow[b]{2}{*}{ Variable } & \multicolumn{4}{|c|}{$\mathbf{A P}$} & \multicolumn{4}{|c|}{ NP } & \\
\hline & Mean & Std. Dev. & Min & Max & Mean & Std. Dev. & Min & $\operatorname{Max}$ & \\
\hline Alcohol [\%/vol] & 14.65 & 0.03 & 14.63 & 14.68 & 13.61 & 0.03 & 13.58 & 13.63 & * \\
\hline Sugar $[\mathrm{g} / \mathrm{L}]$ & 1.60 & 0.07 & 1.52 & 1.66 & 1.42 & 0.07 & 1.35 & 1.48 & $*$ \\
\hline Density [g/L] & 0.99 & 0.00 & 0.99 & 0.99 & 0.99 & 0.00 & 0.99 & 0.99 & $*$ \\
\hline Total dry extract [g/L] & 22.37 & 0.40 & 21.90 & 22.60 & 23.50 & 0.34 & 23.13 & 23.78 & $*$ \\
\hline $\mathrm{pH}$ & 3.29 & 0.02 & 3.27 & 3.30 & 3.46 & 0.01 & 3.45 & 3.47 & $*$ \\
\hline Total acidity [g/L] & 5.63 & 0.04 & 5.60 & 5.67 & 5.84 & 0.04 & 5.79 & 5.86 & $*$ \\
\hline Volatile acidity [g/L] & 0.52 & 0.02 & 0.49 & 0.53 & 0.33 & 0.02 & 0.32 & 0.36 & $*$ \\
\hline Malic acid [g/L] & 0.90 & 0.07 & 0.84 & 0.97 & 1.86 & 0.07 & 1.78 & 1.92 & $*$ \\
\hline Citric acid $[g / L]$ & 0.20 & 0.00 & 0.20 & 0.21 & 0.27 & 0.01 & 0.26 & 0.27 & $*$ \\
\hline Tartaric acid $[\mathrm{g} / \mathrm{L}]$ & 2.71 & 0.05 & 2.68 & 2.77 & 2.35 & 0.05 & 2.30 & 2.40 & $*$ \\
\hline Potassium $[\mathrm{g} / \mathrm{L}]$ & 1.00 & 0.03 & 0.97 & 1.02 & 0.89 & 0.02 & 0.87 & 0.91 & $*$ \\
\hline Glycerine [g/L] & 7.00 & 0.02 & 6.98 & 7.02 & 5.87 & 0.02 & 5.85 & 5.89 & * \\
\hline Ashes $[\mathrm{g} / \mathrm{L}]$ & 1.95 & 0.06 & 1.90 & 2.01 & 2.58 & 0.07 & 2.52 & 2.65 & $*$ \\
\hline OD420 & 1.41 & 0.10 & 1.31 & 1.51 & 0.76 & 0.10 & 0.67 & 0.87 & * \\
\hline OD520 & 1.74 & 0.13 & 1.61 & 1.86 & 1.74 & 0.09 & 1.65 & 1.82 & ns \\
\hline OD620 & 0.52 & 0.03 & 0.49 & 0.56 & 0.47 & 0.04 & 0.43 & 0.51 & ns \\
\hline Polyphenols [mg/L] & 291.33 & 5.69 & 285.00 & 296.00 & 320.67 & 5.03 & 316.00 & 326.00 & $*$ \\
\hline Catechins [mg/L] & 11.57 & 1.23 & 9.00 & 13.00 & 14.60 & 1.56 & 11.90 & 17.00 & * \\
\hline Acetaldehyde [mg/L] & 53.67 & 3.06 & 51.00 & 57.00 & 47.33 & 2.79 & 43.00 & 50.00 & $*$ \\
\hline Methanol [g/L] & 0.04 & 0.00 & 0.04 & 0.04 & 0.03 & 0.00 & 0.03 & 0.03 & * \\
\hline $\mathrm{CO}_{2}[\mathrm{~g} / \mathrm{L}]$ & 650.00 & 10.58 & 638.00 & 658.00 & 620.67 & 9.07 & 611.00 & 629.00 & $*$ \\
\hline Calcium [mg/L] & 39.93 & 0.81 & 39.00 & 40.50 & 63.67 & 1.15 & 63.00 & 65.00 & * \\
\hline Copper [mg/L] & 0.29 & 0.00 & 0.29 & 0.29 & 0.33 & 0.01 & 0.32 & 0.33 & $*$ \\
\hline Sulphates [mg/L] & 0.56 & 0.01 & 0.56 & 0.57 & 0.71 & 0.01 & 0.70 & 0.71 & * \\
\hline
\end{tabular}

AP, air pressing; NP, nitrogen pressing. *Indicates significance at $\mathrm{P} \leq 0.01$ and ns indicates not significant. 
wine with a quality standardisation in a multivariate system like wine and wine fermentation procedures.

PCA was performed to extract composite indicators (components) of the must's characteristics that help to explain differences in the musts obtained by using the two pressing modes.

Table 4 reports all the 15 components extracted from PCA performed on must data. The first column lists the eigenvalues of the correlation matrix, ordered from the largest to the smallest. As we are analysing a correlation matrix, the variables are standardised to have unit variance, so the total variance is 15 . As the eigenvalues are the variances of the principal components, the first principal component has variance 5.583 explaining $37.2 \%(5.583 / 15)$, the second component has variance 5.261 explaining the $35 \%$ of the total variance and so on for the other components. Following the well know and most used Kaiser's (1960) criterion and the scree plot (not shown in the paper) (Cattell, 1966) which suggest to retain components with eigenvalue higher than 1 , three components were extracted that overall explain $82 \%$ $(0.372+0.351+0.096)$ of the total variance of the must components.

The score biplot gives us a feeling for the similarities and differences between the pressing mode in component loading.

The correlation between elements of must and the first principal component extracted suggests us to name it as fermentation component due to its positive correlation with sugars, citric acid, methanol and $\mathrm{CO}_{2}$ and negative correlation with alcohol and RAN. The second component measures antioxidant and preservative characteristics given its correlation with malic acid, polyphenols and ashes and negative correlation with volatile acidity. The third component can measure the must character just its correlation with tartaric acid and $\mathrm{pH}$. Figure 3 shows the biplot that highlights a net separation between the musts obtained with the two pressing modes, AP and NP, in the combination of the three components extracted.

Biplot shows that AP1 (must in the first time of observation) is opposite to NP3. The NP pressing mode has higher antioxidant and preservative characteristics associated with lower fermentation component than AP pressing mode. Biplot of the second and third components that measure the character of must and its stability shows a net separation between the two pressing modes.

The component 3 has a higher correlation both in AP and NP when values are observed in time 2 even if with a net separation between the data of the two pressing modes.

\section{Conclusions}

The application of innovative machines and technologies, as the closed tank pneumatic press with nitrogen use and recovery tested in this study, produced a wine with higher qualitative characteristics than the wine obtained with the traditional pressing performed in presence of oxygen with the same machine.

White musts with higher phenolic content can be obtained by using inert pressing, thereby increasing the nutraceutical properties of the resulting wines. Overall, numerous quality parameters of Sauvignon Blanc must and wine correlate positively with the use of nitrogen during grapes pressing. Moreover, principal component analyses make it possible to extract composite quality indicators of must and wine for evaluating the effectiveness of inert pressing procedure on Sauvignon Blanc grapes.

The results obtained in this study are encouraging and need to be deepened especially with reference to other white grape cultivars.
Table 4. Eingenvalues of the must principal component.

\begin{tabular}{lccc} 
Component & Eigenvalue & $\begin{array}{c}\text { Proportion of } \\
\text { variance } \\
\text { explained }\end{array}$ & $\begin{array}{c}\text { Cumulative } \\
\text { variance } \\
\text { explained }\end{array}$ \\
Comp1 & 5.583 & 0.372 & 0.372 \\
Comp2 & 5.261 & 0.351 & 0.723 \\
\hline Comp3 & 1.438 & 0.096 & 0.819 \\
Comp4 & 0.789 & 0.053 & 0.871 \\
\hline Comp5 & 0.662 & 0.044 & 0.916 \\
Comp6 & 0.514 & 0.034 & 0.950 \\
\hline Comp7 & 0.381 & 0.025 & 0.975 \\
Comp8 & 0.172 & 0.011 & 0.987 \\
\hline Comp9 & 0.068 & 0.005 & 0.991 \\
Comp10 & 0.056 & 0.004 & 0.995 \\
\hline Comp11 & 0.042 & 0.003 & 0.998 \\
Comp12 & 0.025 & 0.002 & 0.999 \\
\hline Comp13 & 0.008 & 0.001 & 1.000 \\
Comp14 & 0.003 & 0.000 & 1.000 \\
\hline Comp15 & 0.000 & 0.000 & 1.000 \\
Total & 15.000 & - & - \\
\hline
\end{tabular}
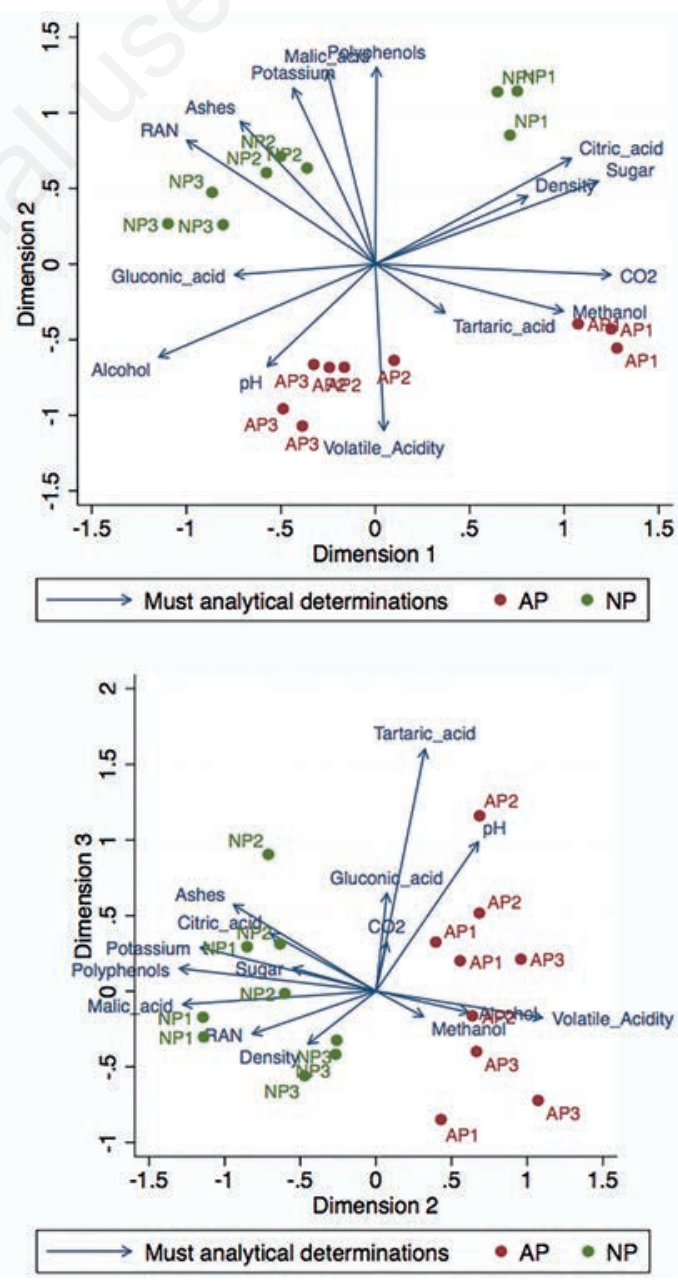

Figure 3. Biplot of must determinations in nitrogen pressing (NP) and air pressing (AP) modes. The subscripts 1, 2 and 3 are referred to the three times of sampling on musts during fermentation (29 ${ }^{\text {th }}$ August, $1^{\text {st }}$ September and $3^{\text {th }}$ September, 2016). 


\section{References}

Boselli E., Di Lecce G., Alberti F., Frega N.G. 2010. Nitrogen gas affects the quality and phenolic profile of must obtained from vacuum-pressed white grapes. LWT- Food Sci. Technol. 43:1494-500.

Catania P., De Pasquale C., Morello G., Vallone M. 2016. Influence of grape transport and destemming systems on the quality of Chardonnay wines. Agric. Eng. Int. CIGR J. 18:2606.

Cattell R.B. 1966. The scree test for the number of factors. Multivar. Behav. Res. 1:245-76.

Cordova A.C., Sumpio B.E. 2009. Polyphenols are medicine: Is it time to prescribe red wine for our patients? Int. J. Angiol. 18:111-7.

De Pasquale F., Siragusa M., Abbate L., Tusa N., De Pasquale C., Alonzo G. 2006. Characterisation of five sour orange clones through molecular markers and leaf essential oils analysis. Sci. Horticult. 109:54-9.

Ferreira-Lima N.E., Burin V.M., Caliari V., Bordignon-Luiz M.T. 2016. Impact of pressing conditions on the phenolic composition, radical scavenging activity and glutathione content of Brazilian Vitis vinifera white wines and evolution during bottle ageing. Food Bioproc. Technol. 9:944-57.

Hartwig P., McDaniel M.R. 1995. Flavor characteristics of lactic, malic, citric and acetic acids at various $\mathrm{pH}$ levels. J. Food Sci. 60:384-8.

Kaiser H.F. 1960. The application of electronic computers to factor analysis. Educ. Psychol. Meas. 20:141-51.

Maggu M., Winz R., Kilmartin P.A., Trought M.C.T., Nicolau L. 2007. Effect of skin contact and pressure on the composition of
Sauvignon Blanc must. J. Agric. Food Chem. 55:10281-8.

Marais J. 1998. Effect of grape temperature, oxidation and skin contact on Sauvignon Blanc juice and wine composition and wine quality. S. Afr. J. Enol. Vitic. 19:10-6.

Mattivi F., Fedrizzi B., Zenato A., Tiefenthaler P., Tempesta S., Perenzoni D., Cantarella P., Simeoni F., Vrhovsek U. 2012. Development of reliable analytical tools for evaluating the influence of reductive winemaking on the quality of Lugana wines. Anal. Chim. Acta 732:194-202.

Motta S., Guaita M., Petrozziello M., Panero L., Bosso A., 2014. Effect of reductive pressing on the concentration of reduced glutathione and phenols in the musts of four Italian cultivars. Am. J. Enol. Vitic. 65:471-8.

Patel P., Herbst-Johnstone M., Lee S.A., Gardner R.C., Weaver R., Nicolau L., Kilmartin P.A. 2010. Influence of juice pressing conditions on polyphenols, antioxidants, and varietal aroma of Sauvignon Blanc microferments. J. Agric. Food Chem. 58:7280-8.

Pons A., Lavigne V., Darriet P., Dubordieu D. 2015. Glutathione preservation during winemaking with Vitis vinifera white varieties: example of Sauvignon Blanc grapes. Am. J. Enol. Vitic. 66:187-94.

Ribéreau-Gayon P., Dubourdieu D., Doneche B., Lonvaud, A. 2000. Handbook of Enology, Volume 1: The microbiology of winemaking and vinifications. Ed. Ribéreau-Gayon P. Wiley, Chichester, England.

Tominaga T., Furrer A., Henry R., Dubourdieu D. 1998. Identification of new volatile thiols in the aroma of Vitis vinifera L. var. Sauvignon Blanc wines, Flavour Frag. J. 13:159-62. 